\title{
Calibration of Aerogel Tiles for the HELIX-RICH Detector.
}

\section{Stephan O'Brien ${ }^{a, *}$ on behalf of the HELIX Collaboration}

(a complete list of authors can be found at the end of the proceedings)

${ }^{a}$ McGill University,

Montreal, QC H3A 2T8, Canada

E-mail: obriens@physics.mcgill.ca

HELIX (High Energy Light Isotope eXperiment) is a balloon-borne instrument designed to measure the chemical and isotopic abundances of light cosmic-ray nuclei. In particular, HELIX is optimized to measure ${ }^{10} \mathrm{Be}$ and ${ }^{9} \mathrm{Be}$ in the range $0.2 \mathrm{GeV} / \mathrm{n}$ to beyond $3 \mathrm{GeV} / \mathrm{n}$. To achieve this, HELIX utilizes a 1 Tesla superconducting magnet with a high-resolution gas drift tracking system, time-of-flight detector, and a ring-imaging Cherenkov (RICH) detector. The RICH detector consists of aerogel tile radiators (refractive index 1.15) with a silicon photomultiplier detector plane. To adequately discriminate between ${ }^{10} \mathrm{Be}$ and ${ }^{9} \mathrm{Be}$ isotopes, the refractive index of the aerogel tiles must be known to a precision of $0.1 \%$. In this contribution, detailed mapping of the refractive index across the aerogel tiles is presented and the methodology used to obtain these measurements is discussed.

$37^{\text {th }}$ International Cosmic Ray Conference (ICRC 2021)

July 12th - 23rd, 2021

Online - Berlin, Germany

\footnotetext{
*Presenter
} 


\section{Introduction}

The High Energy Light Isotope eXperiment (HELIX, [1]) is a balloon-based experiment designed to measure the chemical and isotopic abundances of light cosmic-ray nuclei. In particular, HELIX is optimized to measure ${ }^{10} \mathrm{Be}$ and ${ }^{9} \mathrm{Be}$ in the $0.2 \mathrm{GeV} / \mathrm{n}$ to $>3 \mathrm{GeV} / \mathrm{n}$ energy range. While ${ }^{10} \mathrm{Be}$ and ${ }^{9} \mathrm{Be}$ are both produced at the same time by spallation of heavier nuclei on interstellar protons, ${ }^{10} \mathrm{Be}$ is radioactive and decays with a half-life of $1.4 \mathrm{My} .{ }^{9} \mathrm{Be}$ however, is stable. Measurements of the ratio of ${ }^{10} \mathrm{Be}$ to ${ }^{9} \mathrm{Be}$ therefore provide an excellent opportunity to constrain the propagation times of local cosmic rays. Indeed, measurements of the ${ }^{10} \mathrm{Be}$ to ${ }^{9} \mathrm{Be}$ ratio maybe be used to discriminate between different propagation models.

HELIX utilizes a 1 Tesla superconducting magnet, with a high-resolution gas drift-tracking system to measure particle rigidity. Particle velocities are measured using a time-of-flight (ToF) counter system and a ring-imaging Cherenkov (RICH) detector. The RICH is designed to measure velocities of particles with energies $\gtrsim 1 \mathrm{GeV} / \mathrm{n}$. The HELIX RICH consists of a radiator plane made of $36(6 \times 6)$ high refractive index $(n \sim 1.15)$ hydrophobic aerogel tiles and a SiPM detector plane. Each aerogel tile is $10 \mathrm{~cm} \times 10 \mathrm{~cm}$ in area and approximately $1 \mathrm{~cm}$ thick. These aerogel tiles were produced by $\mathrm{M}$. Tabata [2] using a novel drying technique [3].

To adequately discriminate between ${ }^{10} \mathrm{Be}$ and ${ }^{9} \mathrm{Be}$, the refractive index must be known to a precision of $\Delta n / n \sim O\left(10^{-4}\right)$. Variations in the aerogel refractive index may occur due to local density variations during the drying process. Hence, to achieve the desired discrimination, precise measurements of the refractive index and variations of the refractive index across the aerogel tile are required.

To measure the refractive index of the aerogel, two methods have been utilised. The first method determines the refractive index by measuring the deflection of a laser beam as it transverses the tile at a known incidence angle [4]. The second method measures the refractive index by measuring the Cherenkov ring produced by relativistic particles as they pass through the aerogel. In this contribution we detail measurements made using the latter method.

This contribution is structured as follows. In Section 2 the data acquisition techniques and apparatus are presented. The data reduction and performance of the our data taking technique are also discussed. In Section 3 an example refractive index map is shown and general features are presented. In Section 4 sources of known systematic uncertainty are discussed and a total systematic uncertainty is estimated. Finally, in Section 5 future plans are discussed.

\section{Electron Beam Scan}

\subsection{Data Acquisition}

Precise measurements of the refractive index were made by measuring the Cherenkov ring produced by a beam of $35 \mathrm{MeV}$ electrons. These measurements were performed at the Ionizing Radiation Standards department of the National Research Council in Ottawa [5]. The electron beam produces $2.5 \mu$ s pulses of electrons at a rate of $60 \mathrm{~Hz}$. The energies of these electrons are normally distributed with a mean energy of $35 \mathrm{MeV}$ and $\sigma_{E} \approx 0.4 \%$.

The aerogel tile is mounted in a scanning frame positioned by stepper motors, which provides $\mathrm{X}-\mathrm{Y}$ motion for the tile. Downstream from the aerogel tile, a purpose built CCD-detector board 


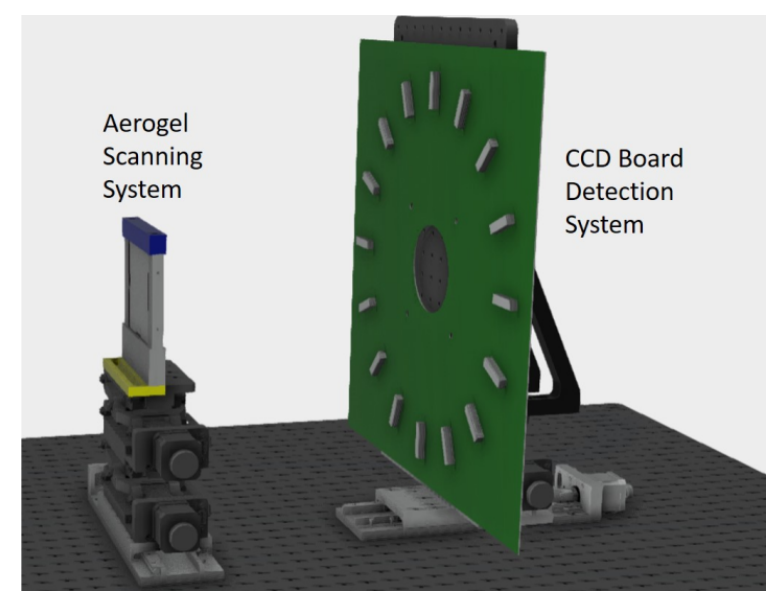

Figure 1: CAD of CCD-detector board and stepper motor system.

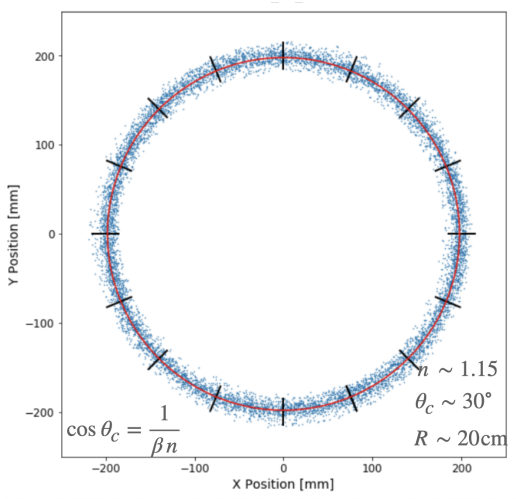

Figure 2: Simulated Cherenkov ring produced by $35 \mathrm{MeV}$ electrons in a $\mathrm{n}=1.15$ radiator. The black lines illustrate the position of each of the $16 \mathrm{CCDs}$, with the red ring showing a fitted circle to the distribution.

(see Figure 1) is used to sample the Cherenkov ring. This CCD-dector board consists of 16 Toshiba TCD1304DG CCDs ${ }^{1}$, arranged in a circle of radius $20 \mathrm{~cm}$ at azimuthal increments of $22.5^{\circ}$ (see Figure 2). Each CCD consists of 3694 pixels with dimension $8 \mu \mathrm{m}$ long by $200 \mu \mathrm{m}$ wide, giving a total active area of each CCD of $29.2 \mathrm{~mm}$ by $0.2 \mathrm{~mm}$. The CCD-detector board is mounted on a system of X-Y-Z control stepper motors to allow for remote re-positioning of the detector.

The face of each aerogel tile is scanned in a $5 \mathrm{~mm}$ grid, with the outer $5 \mathrm{~mm}$ excluded from from the scan. These edge points are excluded from the scan process, as the produced Cherenkov ring would be truncated on one side due to the surrounding aluminium frame. Hence, not providing a symmetric sample of the Cherenkov ring. The aerogel area scanned corresponds to a 19x19 grid spanning 5-95 $\mathrm{mm}$ of the aerogel tile.

At each scan point, 100 "images" are obtained. Each image consist of $16 \mathrm{CCD}$ traces and correspond to a beam pulse of $\sim 10^{10}$ electrons. The images are recorded using a single-board cPCI-6620 series Adlink computer in a crate connected to four Acqiris U1063A DC270 Fast analog-

\footnotetext{
${ }^{1}$ https://toshiba.semicon-storage.com/us/semiconductor/product/linear-image-sensors/ detail. TCD1304DG.html
} 

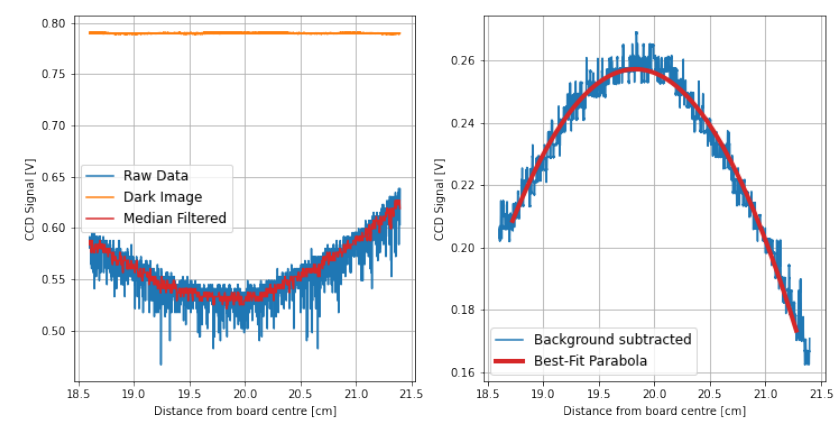

Figure 3: (Left) Observed CCD trace for beam-on data is shown in blue. The median filtered data is shown in red, with the mean background image shown in orange. (Right) Reduced CCD trace with the best-fit parabola shown as a red line.

to-digital Converters (FADCs). The FADCs provide 8 bit resolution and can operate at a sample rate of $1 \mathrm{GS} / \mathrm{s}$. An external trigger for the FADC is provided by a "pre-trigger" signal delivered by the electron beam. This pre-trigger allows for a delayed exposure of the CCDs of $20 \mu \mathrm{s}$. This wide exposure window is centred on the expected arrival time of the electron beam pulse, with ample allowance for timing jitter. The aerogel-CCD system is enclosed within a dark box, to reduce background light levels. The images are recorded to a custom binary format and analysed in near real time $\mathrm{O}(1$ minute), via a "quicklook" analysis. The quicklook analysis is used in conjunction with X-Y-Z stepper motors to centre the CCD-detector board with respect to the Cherenkov ring, prior to each scan.

Prior to data taking, 100 "dark" images are taken. During these exposures, the electron beam is inactive. These are later used to estimate and remove any irreducible background light leakage (e.g. due to emergency lighting). The total scanning process take approximately 30 minutes for a single aerogel tile, with an additional 15 minutes required between scans for changing aerogel tiles.

\subsection{Data Reduction and Analysis}

The data is converted to Flexible image transport system (FITS) format for storage and distribution. For each scan point, all of the 16x100 CCD traces are analysed. The raw CCD traces are shown in Figure 3. A sliding median-filter of widow size 11 pixels, is applied to the data. This reduces statistical pixel-to-pixel variations from the data. As the CCDs measure increasing brightness as a decreasing voltage, the background is removed by subtracting the CCD trace from the time-averaged dark image for the corresponding CCD. The resulting CCD trace shows a broadly peaked distribution that is well-described by a parabola. The peak of this parabola corresponds to the peak of the Cherenkov ring. The broadness of this distribution is due to divergence of the electron beam, multiple scatter of electrons (see Section 5) and aberration coming from the finite thickness of the tile.

The peak of the best-fit parabola is projected into the X-Y plane of the CCD-detector board and a circle is fit to the data, with the $\mathrm{X} / \mathrm{Y}$-center and radius of the circle fit as free parameters.

When determining the Cherenkov angle $\left(\theta_{c}\right)$ one needs to take account of the thickness of the aerogel and the refraction of the Cherenkov emission as it exits the aerogel tile (see Figure 4). 


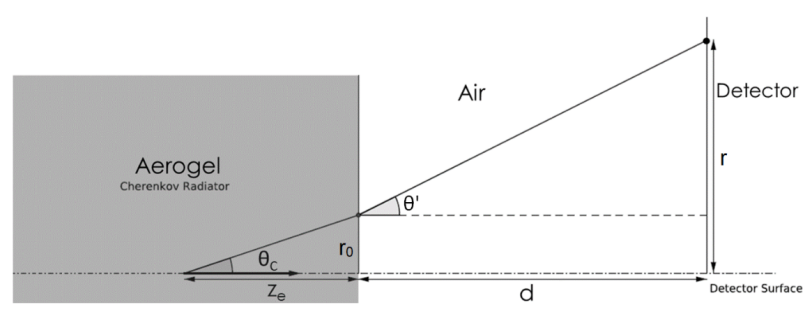

Figure 4: Diagram of Cherenkov emission angle.

The peak of Cherenkov ring approximately corresponds to the light emitted at the half width of the aerogel tile $\left(z_{e}\right) . \theta_{c}$ can be obtained by solving:

$$
\tan \theta_{c}=n_{0} \beta\left(\frac{r-z_{e} \tan \theta_{c}}{\sqrt{\left(r-z_{e} \tan \theta_{c}\right)^{2}+d^{2}}}\right),
$$

where $n_{0}$ is the refractive index of air, $\beta$ is the relativistic velocity of the electrons, $r$ is the Cherenkov ring radius and $d$ is the expansion length of the aerogel-CCD system. The refractive index is given by:

$$
n=\frac{1}{\beta \cos \theta_{c}}
$$

Precise measurements of the aerogel thickness and the relative position of the aerogel tiles within their aluminium frames were made using the Mitutoyo QV 606 coordinate measuring machine (CMM) at TRIUMF. The CMM data is used to apply a correction to $z_{e}$ and $d$ at each scan position.

In a default setup of $d=275 \mathrm{~mm}$ and $35 \mathrm{MeV}$ electron energy, the setup described here can accurately measure a refractive index in the 1.15-1.165 range to a statistical uncertainty of $\Delta n / n \sim O\left(10^{-4}\right)$. Re-positioning of the CCD-detector board using the stepper motor system allows for full coverage of the refractive indices of the HELIX aerogel tiles.

\section{Results}

Refractive index maps are obtained for each of the aerogel tiles. An example of a refractive index map is shown on the left of Figure 5, with the distribution of measured refractive indices shown on the right of Figure 5. The refractive index varies slowly across the face of the aerogel. These variations are greater than the statistical uncertainty of the measured refractive indices themselves and well-described by a $2 \mathrm{D}$ polynomial fit. The tiles typically show a lower refractive index at the centre, with higher refractive indices towards the edges.

Figure 6 shows a slice across the refractive index map. The best-fit 2D polynomial is shown as a black line, with the mean, $68 \%$ and $90 \%$ containment of the tile refractive index distribution shown as solid blue, "dot-dashed" blue and "dashed" red lines, respectively. 

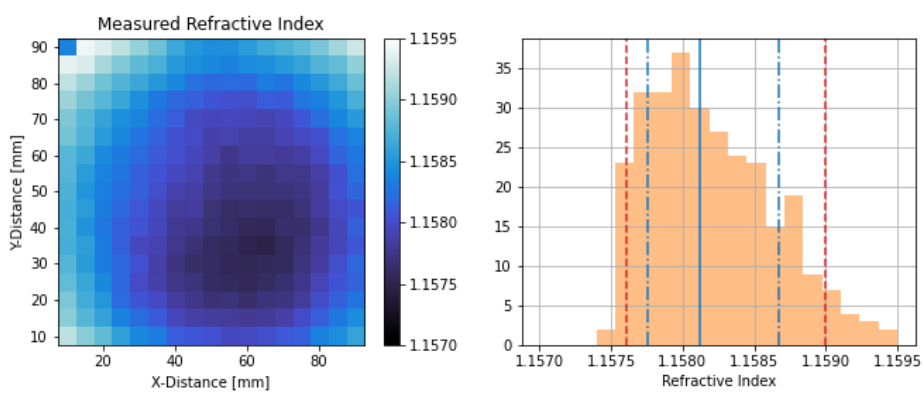

Figure 5: (Left) measured refractive index values via the electron beam method. (Right) Distribution of measured refractive index values. The solid blue line shows the median value. The blue "dot-dashed" and red "dashed" lines correspond to $68 \%$ and $90 \%$ containment of the distribution.

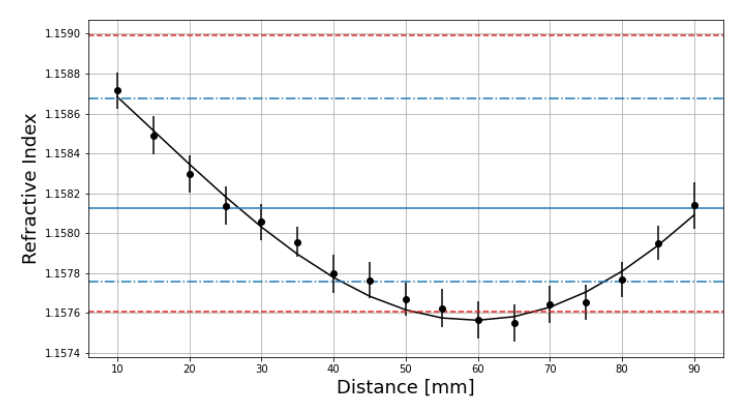

Figure 6: Slice of measured refractive index values across the tile. The black line shows the best-fit 2D polynomial fit to the tile. The solid blue line shows the tile median value . The blue "dot-dashed" and red "dashed" lines correspond to $68 \%$ and $90 \%$ containment of the tile refractive index distribution.

\section{Estimation of Systematic Uncertainties}

Four dominant sources of systematic uncertainty have been identified in this experimental setup. These sources and the corresponding systematic uncertainty are described in Table 1.

\begin{tabular}{|c|c|c|}
\hline $\begin{array}{c}\text { Symbol } \\
(1)\end{array}$ & Description & Estimated uncertainty \\
\hline$\Delta n_{d}$ & Distance from the aerogel to the detector plane & $0.05 \%$ \\
$\Delta n_{t}$ & Thickness of the aerogel & $<0.01 \%$ \\
$\Delta n_{e^{-}}$ & Energy of the incident electrons & $\ll 0.01 \%$ \\
\hline$\Delta n_{\text {sys }}$ & Total systematic uncertainty & $0.06 \%$ \\
\hline
\end{tabular}

Table 1: Systematic uncertainties considered.

The uncertainty on the distance between the downstream aerogel face and the detector plane $\left(\Delta n_{d}\right)$ was determined by measuring the distances between the CCD-detector board's stepper motor at the "home" position and the aerogel mounting frame, using a machined metal rod of known length and known tolerance. The uncertainty in this distance also incorporates the thickness of the CCD PCB board and the aerogel mounting frame, both of which have assigned manufactured 
tolerances. The aerogel's position within the aluminium frame has been measured via a CMM scan performed at TRIUMF. The uncertainty on this measurement is also reflected in $\Delta n_{d}$.

The uncertainty in the aerogel thickness $\left(\Delta_{t}\right)$ is estimated by comparing multiple CMM measurements of the same aerogel tiles. The uncertainty on the thickness is estimated from standard deviation of the scan-to-scan variation. $\Delta_{t}$ affects the effective emission point of the Cherenkov emission, $z_{e}$. As discussed above, the thickness of the aerogel will also vary the expansion length $d$.

The uncertainty on the electron energy $\Delta_{e^{-}}$arises due to the non-uniform energy of the electron. This will affect the relative velocity of the electrons, and therefore the Cherenkov angle $\theta_{c}$. The uncertainty on the electron energy is $0.4 \%$ [5].

The uncertainties are propagated through the analysis chain to estimate their effects on the measured refraction index. The systematic uncertainties for each component are reported in Column (3) of Table 1 . The total systematic uncertainty on the refractive index is obtained via a quadrature sum of the four components and is determined to be $\Delta n_{s y s} \approx 0.06 \%$. This is $\sim 5$ times the statistical error on the refractive index.

\section{Discussion}

In this contribution we have presented a novel method to accurately measure the refractive index of aerogel tiles using a relatively inexpensive array of linear CCDs. This method has been applied to the calibration of HELIX aerogel tiles and has allowed for precise measurements and variations of the refractive index across the aerogel tiles.

Section 4 estimates the systematic uncertainty due to the CCD-board detector to be $\sim 0.06 \%$. Alternative calibration methods are being performed [4], which shall aid in identifying any unseen sources of systematic uncertainty. In addition to this, we are investigating additional systematic effects. A simulation has been developed to study the effects of beam divergence and the aerogel shape on the photon distribution. The results of these studies will be incorporated into a future publication on this calibration process.

\section{References}

[1] Park N. et al., These Proceedings

[2] Tabata M. et al., 2020, Nuclear Instruments and Methods in Physics Research A, 952, 161879. doi:10.1016/j.nima.2019.02.006

[3] Tabata M. et al., 2010, Nuclear Instruments and Methods in Physics Research A, 623, 339. doi:10.1016/j.nima.2010.02.241

[4] Allison P. et al., 2019, ICRC, 36, 133

[5] Ross C.K. at al., (2008), Med. Phys., 35: 4121-4131. 


\section{Full Authors List: HELIX}

P. Allison ${ }^{1}$, J. J. Beatty ${ }^{1}$, L. Beaufore ${ }^{2}$, Y. Chen ${ }^{3}$, S. Coutu ${ }^{3}$, E. Ellingwood ${ }^{4}$, M. Gebhard ${ }^{5}$, N. Green ${ }^{6}$, D. Hanna ${ }^{4}$, B. Kunkler ${ }^{5}$, I. Mognet ${ }^{3}$, R. Mbarek ${ }^{2}$, K. McBride ${ }^{1}$, K. Michaels ${ }^{5}$, D. Müller ${ }^{2}$, J. Musser ${ }^{5}$, S. Nutter ${ }^{7}$, S. O'Brien ${ }^{4}$, N. Park $^{8}$, T. Rosin ${ }^{4}$, E. Schreyer ${ }^{2}$, G. Tarlé6, M. Tabata ${ }^{9}$, A. Tomasch ${ }^{6}$, G. Visser ${ }^{5}$, S. P. Wakely ${ }^{2}$, T. Werner ${ }^{2}$, I. Wisher ${ }^{2}$, and M. Yu ${ }^{3}$

${ }^{1}$ The Ohio State University, Columbus, OH 43210 USA

${ }^{2}$ University of Chicago, Chicago, IL 60637 USA

${ }^{3}$ Pennsylvania State University, University Park, PA 16802 USA

${ }^{4}$ McGill University, Montreal, QC H3A 2T8, Canada

${ }^{5}$ Indiana University, Bloomington, IN 47405 USA

${ }^{6}$ University of Michigan, Ann Arbor, MI 48109 USA

${ }^{7}$ Northern Kentucky University, Highland Heights, KY 41099 USA

${ }^{8}$ Queen's University, Kingston, ON K7L 3N6, Canada

${ }^{9}$ Chiba University, Chiba, 263-8522, Japan 